\title{
The changing pattern of pathology ${ }^{1}$
}

\author{
E. M. DAR MADY
}

Before the inception of the National Health Service not all hospitals in Great Britain had a laboratory service and indeed commercial undertakings and local authorities vied with the clinical pathologists in providing diagnostic services. In pre-war days most provincial clinical pathologists were appointed to a single hospital and were responsible for all branches of pathology, as is still customary in provincial North America, and often local medical officers of health were responsible for the public health aspects of bacteriology such as diagnosis of diphtheria. The laboratory technician was usually a promoted bottle washer without any basic scientific knowledge. Because most pathologists were paid by the hospital they were debarred from the Hospital Medical Committee. It was in this atmosphere that I first entered provincial clinical pathology in 1937. However, the war, the advent of the National Health Service, and the abolition of duplicated laboratory services by the local authority and the commercial firms revolutionized the laboratory services in Great Britain. Perhaps the most noticeable change has been the growth of each discipline of pathology, with the result that it is now impossible for one man to be a master of the entire subject and specialization has become essential to the laboratory. This division had already been established in a number of teaching hospitals, although not all disciplines have yet achieved the equal status of a professorial chair at each teaching hospital.

In the provinces three developments have contributed to this changing scene. First, the formation of the Public Health Laboratory Service; secondly, the setting up of hospital management committees to cover a number of hospitals; third, a demand for increased investigation coupled with the shortage of laboratory accommodation. The Public Health Laboratory Service has not only shown how valuable specialization can be to the community, but also has provided microbiologists of the highest order. The concept of management committees responsible for a number of hospitals has led medical staff to accept appointments to a number of hospitals as a rational method of hospital service.

To try and see the new patterns of pathology more clearly I sent out a questionnaire containing what I hoped were leading questions, and much of what follows is based on the answers I received.

${ }^{1}$ Extracts from the Presidential Address to the Association of Clinical Pathologists, 20 September 1963.
It is perhaps interesting to learn from my questionnaire that there are at least 105 area services already in existence. If this is a representative sample there are probably double that number, for of 657 from 1,365 replies sent to the questionnaire 572 were analyzed. These showed that the majority (456) are in the National Health Service and $\mathbf{1 1 6}$ are associated with universities. This means that about $72 \%$ of our members are in the National Health Service, $20 \%$ are associated with the universities, and about $8 \%$ are engaged in highly specialized work.

I was also anxious to try and determine how far 383 consultant pathologists had been able to specialize in the various disciplines. To my surprise I found that the members who were able to do so were few (146), indeed approximately only onethird of those consultants replying. It is of importance to note that these were confined to hospital laboratories catering for more than 1,200 beds. Of the remainder, 71 had to be master of four or more disciplines, 10 of three, and 156 of two. This might suggest to the Examining Committee of the College of Pathologists that a final examination in a number of disciplines may still be desirable.

The recent discussion on the Ministry of Health building note no. 15 and the Platt Committee enquiry has also made me wonder what criterion can be applied to assess the proper number of pathologists for any particular area. In many specialities the numbers of consultants can be related to the catchment area or population and possibly to the number of hospital beds. In pathology many more variables would seem to operate, those, in my opinion, being the number of hospital beds, the catchment population, the number of clinical consultants, junior staff, and general practitioners. As the result of the replies I have tried to determine if there is any relationship between the number of pathologists and these five variables.

It is usually accepted that a new district hospital of 800 beds will provide a service to a population of approximately 250,000 . It is, therefore, of interest to know whether at present pathologists of whatever speciality are serving this number of beds or this population.

The majority of pathologists serve more than 800 beds: indeed over 50 (probably double that number in the Association) serve 1,200 to 2,000 and nearly 40 more than 2,000 beds. However, the population 
covered is rather more problematical since it would seem that an almost equal number of pathologists serves populations from 150,000 to 350,000 . I would again reiterate that none of the pathologists who were able to specialize in a single discipline was attached to hospitals of 1,200 beds but more often to hospitals of over 2,000 beds. If we look at this in a different way, and if we accept that the work load from each discipline from a hospital of 800 beds was equal, then we might expect to find that the number of pathologists in each discipline would be equal. But, if the work load were unequal, then the number of pathologists practising each discipline would be unequally distributed. I must add that the problem is slightly obscured by the fact that a number of pathologists have to practise more than one discipline.

Today although there are more pathologists practising morbid anatomy than haematology, the greatest number serve between 1,200 and 2,000 beds, suggesting that the haematologists find the area they can cover is smaller than that of the morbid anatomists, whereas the bacteriologist has to look after an area larger than either haematologist or morbid anatomist. The number of chemical pathologists is small in comparison with the pathologists of the three other disciplines. This can be accounted for by the fact that a number of nonmedical biochemists have not been recorded.

It is often said that members of the junior staff of a hospital are responsible for unnecessary work of the laboratory, but in fact the influence of consultant and junior staff is in proportion to one another. Each pathologist seems to be serving about 30 to 50 consultants and a similar number of junior staff, and thus this statement cannot be regarded as true.

So far I have not considered the pattern of the group or area laboratory. There appear to be two distinct patterns. First, there are those laboratories which cover four to six hospitals with up to 800 beds and employing one to 30 technicians and three pathologists; second, the laboratories which cover nine or more hospitals with more than 2,000 beds and employ 41-60 technicians and nine or more pathologists. Again I would make the point that only in those laboratories which cover more than 1,200 and up to 2,000 beds at present provide a fully specialized service split into the four disciplines.

\section{LABORATORY SERVICES FOR THE GENERAL PRACTITIONER}

The impact of the general practitioner upon the laboratory cannot be said to be great since it is doubtful if his requests ever exceed $10 \%$ of the total. The type of investigation requested by the general practitioner differs considerably from that of the consultant due to the fact that a large number of patients consult their practitioners for psychologicaf reasons and his first concern is to exclude organice disease. Therefore, the requests for investigations submitted from him are likely to be aimed aह excluding disease rather than proving it. This provides an entirely different and perhaps mores difficult challenge to the laboratory than th 5 investigations asked for by the hospital staff: It is also interesting to note how much variatiorw there is in the type and number of requests which come from consultants and general practitioners.

The resulting demands for pathological investi $\vec{v}$ gations have led to an increase in the number of pathologists in any one laboratory, and to thet division of the laboratory into the four main disciplines. Indeed the pattern of investigation in any particular disease varies from doctor to doctor $\vec{\varphi}$ To show the size and complexity of the problems I have carried out a survey of the requests sent toके us from the general practitioners, excluding locums and assistants. The names of the doctors werep obtained from the appropriate executive councilst of the area and compared with those submitting requests to the laboratory. In 1962 there were 238 general practitioners who submitted 27,445 requests, an average of 115 requests per doctor Five general practitioners sent no specimens at allo 10 sent us fewer than 10 , and 30 fewer than 30 . AD the other end of the scale six sent us between 300 and 400 specimens, one between 400 and 500 , and tw $\overrightarrow{5}$ more than 500. At first sight it is difficult to explain this variation. However, in order to examine the position in more detail, I have divided the practition? ers into six categories: (1) those who run their own hospital and are largely responsible fo? treating their own patients when in hospital; (2 those whose patients are largely from the professe ional classes and live on the outskirts of the City (3) those who run a mixed practice containing aff categories of patients; (4) those whose patients are largely from Council estates; (5) those whose patients live in the centre of the City of Portsmouth and (6) those whose patients come from rural of remote areas or where communications are difficult.

Those general practitioners who run their owfis hospitals provide the most work with a mean of 224 requests in the year. Close behind are the doctof? whose patients are largely of the professional classe whilst those in the Council estates and the City of Portsmouth (the main hospitals and Centrap Laboratory are within the City) submit less than haf those from the first two categories, 99 and 92 respectively. It is perhaps of interest that three out \& 
five doctors sent us no specimens and half of those who sent fewer than 10 came from the City. It is also noticeable that the doctors whose patients provided 314 requests from the City of Portsmouth were largely drawn from professional classes. It is possible that in the sixth, practitioners from remote or rural areas of Hampshire, may send requests to neighbouring area laboratories. These facts are analysed in Table I.

\begin{tabular}{l}
\multicolumn{3}{c}{ TABLE I } \\
\\
G.P.s'
\end{tabular}

I was interested to know whether, as the result of these investigations, there was any difference in the number of patients referred for out-patient consultations. As the populations vary in each locality $\mathbf{I}$ decided to express this as the number of patient attendances per 1,000 of population in each area for the year 1962. These figures were fortunately known as they had been prepared for another purpose and are shown in the final column of Table I. Only 5 patients per 1,000 of the first two categories are referred to hospitals as compared with five times that number from mixed practices, and more than seven from the City of Portsmouth and 10 times those living in Council estates. It is, however, possible that patients in the first two categories may be seen by consultants in private or are referred to consultants at adjacent groups or in London. In spite of this, however, the inverse relationship of the number of investigations and the number of referrals in each category does suggest that the doctors who investigate their own cases reduce the number of consultations at out-patient departments.

One further point of interest arising from the survey was the proportion of work (divided into disciplines) which was provided by the general practitioners and the out-patient and in-patient departments of the hospitals. This information might be of some value when setting up a new laboratory service.

A large proportion of the requests for investigation from general practitioners were for haematological and ante-natal tests (Table II). In fact, the
TABLE II

REQUESTS FOR LABORATORY TESTS FROM GENERAL PRACTITIONERS

\begin{tabular}{lccc} 
& $\begin{array}{l}\text { General } \\
\text { Practitioners }\end{array}$ & Out-patients & In-patients \\
\hline Haematology & $47 \%$ \\
Ante-natal & $26.5 \%$ & $54 \%$ & $44.4 \%$ \\
Bacteriological & $16.6 \%$ & $33.2 \%$ & $26.6 \%$ \\
Biochemistry & $9.7 \%$ & $8.5 \%$ & $21.9 \%$ \\
Histology & - & $24.3 \%$ & $6.1 \%$ \\
Necropsies & - & $1.9 \%$ & $1.0 \%$
\end{tabular}

volume of haematological tests for general practitioners was greater than those arising from out-patient departments, although in fact the technical complexity of the work cannot be regarded as high as that required for in-patients and out-patients. This is confirmed by the fact that $80 \%$ of the results of haematological investigations from general practitioners were within normal accepted limits.

\section{MORBID ANATOMY}

One of the most controversial matters in trying to assess the need for more morbid anatomists is the number of necropsies that pathologists should perform per day. It is therefore interesting to note that the numbers performed individually vary considerably, although the majority of pathologists do fewer than 100 necropsies annually. At the other end of the scale, 41 state that they carry out between 250 and 350, 24 between 451 and 550, three between 551 and 750 , and three more than 750 annually. It is also perhaps surprising that those who exceed 350 stated that they were responsible for surgical biopsies as well as forensic pathology, and that of 228 consultants practising morbid anatomy, 136 also undertook forensic pathology. I believe this confirms the practical difficulties of setting up an independent forensic pathology service. These facts are of interest for another reason and concern the recognition of a laboratory for training, for the American College of Pathology will not recognize laboratories which carry out fewer than 2,000 biopsies and fewer than 75 necropsies annually. If these criteria were applied here the majority of laboratories (nearly 80 ) would not be accepted for training, and one wonders too whether this volume of work is sufficient to maintain a satisfactory standard.

\section{MICROBIOLOGY}

During the past 15 years the recognition of hospital cross infection has increased and new methods for sterilizing instruments and dressings have been introduced. The microbiologist has had to leave the bench to undertake duties such as that of a 
cross infection officer and in many instances he has taken on the role of medical superintendent. The answers to my questionnaire confirmed this, for of 187 consultants who replied to this question, 167 are responsible for testing sterilizers, six of whom have 17 or more under their wing. This is no mean task even if sterilizers are working satisfactorily.

There is another interesting sidelight which concerns microbiology. From the figures obtained from the questionnaire about 5,000 urine samples are tested bacteriologically daily. In spite of protests catheters are still widely used to obtain specimens for this purpose. In our laboratories about half the urines are from women. We have also established that about $10 \%$ of the catheters 'sterilized' by boiling on the wards grow pathogenic organisms. These figures suggest that in this country about 2,500 women alone had been subjected daily to the subsequent risk of pyelonephritis, while a further 250 are likely to have living organisms introduced into the bladder. This is in itself a sobering thought and it is time that the country was made aware of the risks that patients are running.

\section{BIOCHEMISTRY}

I had a number of indignant letters saying that the number of electrolyte estimations performed daily was no way to judge the output of a laboratory, with which statement I would whole-heartedly agree. In our experience the results of performing electrolytes in batches of less than 10 are often unreliable. Sixty laboratories performed less than five, 45 less than 15 , and only 38 performed 15 or more while eight performed 46 or more. This suggests that in the smaller laboratory unacceptable technical errors may occur and I would like to produce evidence which I think substantiates this statement. A few years ago it was our practice to carry out most of the work at the main or central laboratory where we normally performed estimations of approximately 20 electrolytes, 40 ureas and sugars daily, whilst at the three smaller laboratories the numbers rarely exceeded 10 daily. The technicians performing the tests had a considerable period of training at the Central Laboratory before undertaking work at smaller laboratories. At the Central Laboratory the tests were done in batches by a technician who usually had only four or so different kinds of tests to do daily, whereas at the smaller laboratories the technician was responsible for approximately the same number of tests but they were varying and of a different complexity. From time to time the technicians were exchanged with those at the Central Laboratory but generally those at the smaller laboratories were of higher seniority than those at the Central Laboratory. In order to test reproducibility and technical accuracy of each $\vec{B}$ laboratory, samples of which I knew the strength were performed simultaneously at all laboratories orf the same type of equipment. The tests investigated were blood ureas, sugars, total proteins, and chlor ides. Our findings showed that although the Centraf Laboratory used quite junior technicians for these estimations, they were able to keep within the accepted technical error of $10 \%$ for blood sugars and ureas and $1.5 \mathrm{mEq} . / 1$. for chlorides. The smallef laboratories showed considerably greater errors frequently $20 \%$ or more for sugars and ureas and $3.0 \mathrm{mEq}$./1. for chlorides. Such technical errors from $\vec{N}$ smaller laboratories could be disastrous in urear clearance or sugar tolerance tests.

More recently we have been using a four-channeb autoanalyzer for these investigations and have now centralized the biochemical work for the hospitals incs the area in one laboratory. We have found thaf the technical error can now be reduced to less than $5 \%$ for sugars and ureas, although chlorides remain at $1.5 \mathrm{mEq}$./l., performing 40 estimations per hour, $\stackrel{\mathbb{Q}}{-}$ but we anticipate that with new flow cells even this technical error can be reduced. This is certainly? superior to anything we could do by hand, although the same class of technician is responsible for the work. Even at 60 per hour the reproducibility of chlorides is as satisfactory as formerly. This makes me wonder whether more centralization of bio응 chemistry and more automation is not now a necessity

\section{MEMBERSHIP OF LEARNED SOCIETIES}

One of the great problems of training of patho logists is the wide range of subjects to which he may be allied and to test this hypothesis I askedo for the number of learned societies to which we as pathologists belong. The majority of clinicab pathologists belong to three to five societies buB some to as many as 15 . Indeed one member stateso that he belongs to more than 16. This confirms how necessary it has become for a pathologist tog have a wide knowledge of medicine, but does it also mean that inadequacies in the scientific side of oup Association have resulted in the birth of a number of splinter group scientific societies?

\section{DISCUSSION}

Unfortunately space does not permit me to include all analyses of the replies to the questionnaire; indeed some were so equivocal that $I$ felt tha 5 answers might be misleading. On the other hand it is clear that the pattern of pathology service which is emerging is one that is not confined to one hospitalo 
and quite clearly covers a larger number of beds and population than the Ministry of Health envisages.

It also appears that at the moment only one third of pathologists are able to practice one discipline and answers suggest, that in order to achieve this ideal, catchment areas of more than 250,000 persons and more than 1,200 beds are required. It is also clear that the work load per pathologist in each discipline is not equal and the figures suggest that it will be necessary to provide more haematologists than morbid anatomists. It would also seem that there are a large number of pathologists who examine fewer than 2,000 surgical biopsies and fewer than 100 cadavers. One wonders whether this volume of work is adequate to ensure that the pathologist can recognize the unusual and has sufficient experience to provide a competent answer. This poses the question as to whether the adjacent group laboratory service of inadequate catchment area should be enlarged to cover more than one management committee, and whether reference laboratories should be set up in a similar way to those formed by Sir Graham Wilson for the Public Health Laboratory Service. It is clear that each area service has grown up in a haphazard manner without thought either by regional hospital boards or by pathologists themselves.

The questions I have posed I believe are as yet unanswered and it is for this reason that I think the time has come when this Association should set up a committee to study the future pattern of pathology. I also believe that some of the differences found in the method of investigating cases arise because the teaching of clinical pathology both to the future practitioners and to clinicians is inadequate. Much more emphasis must be placed on how organic disease can be excluded. I believe that the example at Portsmouth and Isle of Wight shows that the general practitioner who investigates his own cases saves the consultants unnecessary out-patient consultations, thus allowing him more time to devote to the more difficult problem of clinical medicine.

In conclusion I would like to thank the members for their kindness in completing the questionnaire and for writing to me. I am also grateful to my colleagues in Portsmouth and the Isle of Wight area for their help and for allowing me access to their records, and to the Portsmouth Group Hospital Management Committee for providing funds in order to analyse the requests from general practitioners, to the Records Officer, Mr. T. W. Thomas, and Mrs. M. Phillips for carrying it out. I would also like to acknowledge the help and cooperation from those at the Wessex Regional Hospital Board Machine Accounting Unit, in particular Mr. J. Heanes, Mr. L. O'Connor, Mr. T. W. Bennion, and Mr. R. Leyton. 